\title{
The impact of the Chebyshev collocation method on solutions of the time-fractional Black-Scholes
}

\author{
H. Mesgarani ${ }^{1}$ - A. Beiranvand ${ }^{1} \cdot$ Y. Esmaeelzade Aghdam ${ }^{1}$
}

Received: 5 July 2020 / Accepted: 22 October 2020 / Published online: 10 November 2020

(c) The Author(s) 2020

\begin{abstract}
This paper presents a numerical solution of the temporal-fractional Black-Scholes equation governing European options (TFBSE-EO) in the finite domain so that the temporal derivative is the Caputo fractional derivative. For this goal, we firstly use linear interpolation with the $(2-\alpha)$-order in time. Then, the Chebyshev collocation method based on the second kind is used for approximating the spatial derivative terms. Applying the energy method, we prove unconditional stability and convergence order. The precision and efficiency of the presented scheme are illustrated in two examples.
\end{abstract}

Keywords Time-fractional Black-Scholes equation - Collocation method · Chebyshev polynomials of the second kind . Linear interpolation $\cdot$ Convergence order and rate $\cdot$ Stability

Mathematics Subject Classification $41 \mathrm{~A} 50 \cdot 91 \mathrm{G} 80 \cdot 34 \mathrm{~K} 37 \cdot 97 \mathrm{~N} 50 \cdot 97 \mathrm{~N} 40$

\section{Introduction}

The Black-Scholes model (BSM) is a mathematical equation for pricing and options treaty. In special, the model computes the transformation over time of financial mechanisms (such as stocks or futures). It presumes that they will have a lognormal distribution of prices. Using this presumption and factoring in other great variables, the model concludes the value of a call option. In 1973, pricing options have experienced a lot of consideration that the first time Black and Scholes [1] and Merton [2] proposed BSM for them. Although this model is very favorite, it has some deficiencies like lacking the "volatility smile"[3] in the actual marketplaces. A strong tool for generalization of this model is the use of fractional derivatives and integrals due to its non-local nature [4]. The concept and applications of fractional calculus have greatly expanded over the nineteenth and twentieth

H. Mesgarani

Hmesgarani@sru.ac.ir

A. Beiranvand

asmabeiranvand1981@gmail.com

Y. Esmaeelzade Aghdam

Yonesesmaeelzade@gmail.com

1 Department of Mathematics, Shahid Rajaee Teacher

Training University, Tehran, Iran centuries, and numerous authors have provided descriptions for fractional derivatives. One of the most popular fractional derivatives is the left and right Caputo derivative of any arbitrary order, real or complex, which is defined as

$$
\begin{aligned}
& { }_{a} \mathcal{D}_{t}^{\alpha} u(x, t)= \\
& \frac{1}{\Gamma(n-\alpha)} \int_{a}^{t}(t-\tau)^{n-\alpha-1} \frac{\partial^{n} u(x, \tau)}{\partial \tau^{n}} d \tau, n-1<\alpha<n, \\
& { }_{t} \mathcal{D}_{b}^{\alpha} u(x, t)= \\
& \frac{(-1)^{n}}{\Gamma(n-\alpha)} \int_{t}^{b}(\tau-t)^{n-\alpha-1} \frac{\partial^{n} u(x, \tau)}{\partial \tau^{n}} d \tau, n-1<\alpha<n,
\end{aligned}
$$

respectively. In addition, taking $\alpha \rightarrow n$, leads to $\lim _{\alpha \rightarrow n} \mathcal{D}_{t}^{\alpha} u(x, t)=\frac{\partial^{n} u(x, t)}{\partial t^{n}}$ and $\lim _{\alpha \rightarrow n} \mathcal{D}_{b}^{\alpha} u(x, t)=\frac{\partial^{n} u(x, t)}{\partial t^{n}}$. There are many ways to solve fractional differential equations such as finite difference methods [5, 6], finite element methods [7], finite volume methods [8], spectral methods [9, 10], and meshless methods [11]. In recently, many authors applied the various methods for solving these equations [12-14].

The detection of the fractional form of the stochastic process led to the discovery of fractional calculus into financial theory. In 2000, a time-fractional BSM was presented by Wyss [15] for the first time. In this article, we consider the presented equation by Wyss, which is as follows 
${ }_{0} \mathcal{D}_{t}^{\alpha} u(x, t)=\rho \frac{\partial^{2} u(x, t)}{\partial x^{2}}+\mu \frac{\partial u(x, t)}{\partial x}-r u(x, t)+f(x, t)$,

$0<x<1, \quad 0<t \leq T, \quad 0<\alpha \leq 1$,

with the following initial and boundary conditions

$u(x, 0)=\psi(x), \quad 0<x<1$,

$u(0, t)=\varphi_{0}(t), \quad u(1, t)=\varphi_{1}(t), \quad 0<t \leq T$,

where $\rho=\frac{1}{2} \sigma^{2}>0, \mu=r-\rho$ and $r>0$ are the known constant and $f(x, t)$ is the source term. When $\rho>0, \mu=0, r \neq 0$ and $\rho>0, \mu<0, r=0$, the model (1) is a reaction-diffusion and time-fractional advection-diffusion model, respectively.

There is even more method for determining the analytical process as [16-19]. Because it is difficult to receive an accurate solution, different approaches to estimate them are presented in [20-25]. In recent years, a finite-difference schema in second-order accurate and an implicit finite differ-
Lemma 1 (See [31]) The Caputo derivative for $0<\alpha \leq 1$ is discretized by the linear model as

${ }_{0} \mathcal{D}_{t}^{\alpha} u(x, t)=\frac{\delta \tau^{-\alpha}}{\Gamma(2-\alpha)} \sum_{j=0}^{M} \lambda_{M, j} u\left(x, \tau_{j}\right)+\mathcal{O}\left(\delta \tau^{2-\alpha}\right)$,

where $\tau_{j}=j \delta \tau, j=0,1, \ldots, M$ is time nodes with the step size $\delta \tau=\frac{T}{M}$ and

$\lambda_{M, j}= \begin{cases}1, & j=M, \\ (M-j-1)^{1-\alpha}-2(M-j)^{1-\alpha}+(M-j+1)^{1-\alpha}, & 1 \leq j<M, \\ (M-1)^{1-\alpha}-(M)^{1-\alpha}, & j=0 .\end{cases}$

Let us that we denote $u^{j}=u\left(x, t_{j}\right), \bar{\rho}=\Gamma(2-\alpha) \rho, \bar{\mu}=$ $\Gamma(2-\alpha) \mu, \mathfrak{r}=r \Gamma(2-\alpha), F^{M}=\Gamma(2-\alpha) f\left(x, t_{M}\right) \quad$ a n d $\bar{\lambda}_{M, j}=-\lambda_{M, j}$, then a semi-discrete scheme of Eq. (1) will be achieved by applying the Lemma 2 as

$\left(\lambda_{M, M}+\mathfrak{r} \delta \tau^{\alpha}\right) u^{M}-\bar{\rho} \delta \tau^{\alpha} \frac{\partial^{2} u^{M}}{\partial x^{2}}-\bar{\mu} \delta \tau^{\alpha} \frac{\partial u^{M}}{\partial x}=\sum_{j=0}^{M-1} \bar{\lambda}_{M, j} u^{j}+\delta \tau^{\alpha} F^{M}+\delta \tau^{\alpha} \mathcal{R}^{M}$,

ence model with first-order is used for solving TFBSE-EO in [26] and [27], respectively. In addition, an explicit-implicit numerical method is proposed by Bhowmik In 2014 to solve the partial integrodifferential equation [28] which is the base of the option pricing hypothesis. In [29], Chen evaluated to price American options with using a predictor-corrector. In 2016, Zhang proposed a discrete implicit numerical scheme for pricing American options [30].

The outline of this paper is organized as below: In Sect. 2, by using linear interpolation for the time variable we get the semi-discrete design of the TFBSE-EO. So, we will consider the stability and convergence analysis. In Sect. 3, we apply a Chebyshev collocation method of the second kind for approximating the spatial fractional derivative for obtaining the full scheme. Finally, the fourth section contains two numerical examples to show the efficiency of the method.

\section{The investigated analysis of the temporal-discrete scheme}

In this section, we first present some notations and define the functional space with the $L_{2}$-norm, $\|u(x)\|_{2}=\langle u(x), u(x)\rangle^{\frac{1}{2}}$, as following that has used in our paper.

$\mathcal{H}_{\Omega}^{n}(\varphi)=\left\{\varphi \in L^{2}(\Omega), \quad \frac{\partial^{\alpha} \varphi}{\partial x^{\alpha}} \in L^{2}(\Omega), \forall|\alpha| \leq n\right\}$,

where $L^{2}(\Omega)$ is the space of measurable function with the square of Lebesgue integrable in $\Omega$. where $C$ is a positive constant such that $\mathcal{R}^{M} \leq C \mathcal{O}\left(\delta \tau^{2-\alpha}\right)$. After eliminating $\mathcal{R}^{M}$ in the mentioned relation, the following semi-discrete scheme can be received

$\left\{\begin{array}{l}\left(\lambda_{M, M}+\mathfrak{r} \delta \tau^{\alpha}\right) U^{M}-\bar{\rho} \delta \tau^{\alpha} \frac{\partial^{2} U^{M}}{\partial x^{2}}-\bar{\mu} \delta \tau^{\alpha} \frac{\partial U^{M}}{\partial x}=\sum_{j=0}^{M-1} \bar{\lambda}_{M, j} U^{j}+\delta \tau^{\alpha} F^{M}, \\ U^{0}(x)=\psi(x), \quad 0<x<1, \\ U^{j}(0)=\varphi_{0}\left(t_{j}\right), \quad U^{j}(1)=\varphi_{1}\left(t_{j}\right), \quad j=0,1, \ldots, M,\end{array}\right.$

where $U^{j}, j=0,1, \ldots, M$ is the approximate solution of Eq. (5).

It is necessary to prove the following lemma to find the convergence order and denote the unconditional stability of the semi-discrete scheme.

Lemma 2 Let $U^{k} \in \mathcal{H}_{\Omega}^{2}, k=1,2, \ldots, M$ and $U^{0}$, be the solution and the initial condition of the semi-discrete (6), respectively, then we have

$\left\|U^{k}\right\| \leq C\left(\left\|U^{0}\right\|+\max _{0 \leq l \leq M}\left\|F^{l}\right\|\right)$,

where $C$ is a positive value.

Proof Multiplying relation (6) by $v \in \mathcal{H}_{\Omega}^{2}$ and then integrating, we conclude 


$$
\begin{aligned}
& \left(\lambda_{M, M}+\mathfrak{r} \delta \tau^{\alpha}\right)\left\langle U^{M}, v\right\rangle-\bar{\rho} \delta \tau^{\alpha}\left\langle\frac{\partial^{2} U^{M}}{\partial x^{2}}, v\right\rangle-\bar{\mu} \delta \tau^{\alpha}\left\langle\frac{\partial U^{M}}{\partial x}, v\right\rangle \\
& =\sum_{j=0}^{M-1} \bar{\lambda}_{M, j}\left\langle U^{j}, v\right\rangle+\delta \tau^{\alpha}\left\langle F^{M}, v\right\rangle .
\end{aligned}
$$

The second and third terms on the left side of the above relation can be written as $\left\langle\frac{\partial^{2} U^{1}}{\partial x^{2}}, U^{1}\right\rangle=-\left\langle\frac{\partial U^{1}}{\partial x} \frac{\partial U^{1}}{\partial x}\right\rangle$, $\left\langle\frac{\partial U^{1}}{\partial x}, U^{1}\right\rangle=0$, respectively and $\lambda_{M, M}=1$. Then, we get

$\mathfrak{r} \delta \tau^{\alpha}\left\langle U^{M}, v\right\rangle \leq\left(\lambda_{M, M}+\mathfrak{r} \delta \tau^{\alpha}\right)\left\langle U^{M}, v\right\rangle \leq \sum_{j=0}^{M-1} \bar{\lambda}_{M, j}\left\langle U^{j}, v\right\rangle+\delta \tau^{\alpha}\left\langle F^{M}, v\right\rangle$.

Now, we apply the mathematical induction on $M$. For $M=1$ and denote $v=U^{1}$ in Eq. (8), we get

$\mathfrak{r} \delta \tau^{\alpha}\left\langle U^{1}, U^{1}\right\rangle \leq \bar{\lambda}_{1,0}\left\langle U^{0}, U^{1}\right\rangle+\delta \tau^{\alpha}\left\langle F^{1}, U^{1}\right\rangle$.

Handling the Cauchy-Schwarz inequality and $\bar{\lambda}_{1,0} \leq 1$ so that we can write the following relation

$\left\|U^{1}\right\| \leq \frac{1}{\mathfrak{r} \delta \tau^{\alpha}}\left\|U^{0}\right\|+\frac{1}{\mathfrak{r}}\left\|F^{1}\right\| \leq C\left(\left\|U^{0}\right\|+\max _{0 \leq l \leq M}\left\|F^{l}\right\|\right)$.

Suppose for $j=1,2, \ldots, M-1$ the below equation is correct

$\left\|U^{j}\right\| \leq C\left(\left\|U^{0}\right\|+\max _{0 \leq l \leq M}\left\|F^{l}\right\|\right)$.

By using the relation (9), one can express the above relation as

$\mathfrak{r} \delta \tau^{\alpha}\left\|U^{M}\right\| \leq C\left(\sum_{j=0}^{M-1} \bar{\lambda}_{M, j}\left\|U^{0}\right\|+\max _{0 \leq l \leq M}\left\|F^{l}\right\|\right)+\delta \tau^{\alpha}\left\|F^{M}\right\|$.

We know from [13] that $1<\sum_{j=0}^{M-1} \bar{\lambda}_{M, j}<2$. Thus, we can conclude

$\left\|U^{M}\right\| \leq C\left(\left\|U^{0}\right\|+\max _{0 \leq l \leq M}\left\|F^{l}\right\|\right)$.

Therefore, the proof of Lemma 2 is completed.

Theorem 1 The achieved semi-discrete scheme (6) by the linear interpolation of the Lemma 2 is unconditionally stable.

Proof Suppose the approximation solution and the initial condition of Eq. (6) be $U^{M} \in \mathcal{H}_{\Omega}^{2}$ and $U^{0}$, respectively. Then, the error $\varepsilon^{j}=u^{j}-U^{j}, j=0,1, \ldots, M$ satisfies as following

$$
\begin{array}{r}
\left(1+\mathfrak{r} \delta \tau^{\alpha}\right)\left\langle\varepsilon^{M}, \varepsilon^{M}\right\rangle-\bar{\rho} \delta \tau^{\alpha}\left\langle\frac{\partial^{2} \varepsilon^{M}}{\partial x^{2}}, \varepsilon^{M}\right\rangle \\
-\bar{\mu} \delta \tau^{\alpha}\left\langle\frac{\partial \varepsilon^{M}}{\partial x}, \varepsilon^{M}\right\rangle=\sum_{j=0}^{M-1} \bar{\lambda}_{M, j}\left\langle\varepsilon^{j}, \varepsilon^{M}\right\rangle .
\end{array}
$$

Using the Lemma 2 and the above relationship, we get
$\left\|\varepsilon^{M}\right\| \leq C\left\|\varepsilon^{0}\right\|$

This completes the condition of unconditional stability.

Theorem 2 Suppose $U^{k}$ and $u^{k}$ for $k=1,2, \ldots, M$ be the approximate solution and the exact of relation (6) and (1), respectively with initial condition $U^{0}=\psi(x)$. Then, we have the following error evaluation

$\left\|u^{k}-U^{k}\right\| \leq C\left\|\mathcal{O}\left(\delta \tau^{2-\alpha}\right)\right\|$,

where $C$ is a positive constant.

Proof Let $\varepsilon^{k}=\left|u^{k}-U^{k}\right|, k=1,2, \ldots, M$ be the error term. We can indeed inscribe the following error equation

$$
\begin{aligned}
(1+ & \left.\mathfrak{r} \tau^{\alpha}\right)\left\langle\varepsilon^{k}, \varepsilon^{k}\right\rangle-\bar{\rho} \delta \tau^{\alpha}\left\langle\frac{\partial^{2} \varepsilon^{k}}{\partial x^{2}}, \varepsilon^{k}\right\rangle-\bar{\mu} \delta \tau^{\alpha}\left\langle\frac{\partial \varepsilon^{k}}{\partial x}, \varepsilon^{k}\right\rangle \\
& =\sum_{j=0}^{k-1} \bar{\lambda}_{k, j}\left\langle\varepsilon^{j}, \varepsilon^{k}\right\rangle+\delta \tau^{\alpha}\left\langle\mathcal{R}^{k}, \varepsilon^{k}\right\rangle,
\end{aligned}
$$

where $\mathcal{R}^{M} \leq C \mathcal{O}\left(\delta \tau^{2-\alpha}\right)$. By considering Lemma 2 and $\left\|\varepsilon^{0}\right\|=0$, we get

$\mathfrak{r} \delta \tau^{\alpha}\left\|\varepsilon^{k}\right\| \leq \delta \tau^{\alpha}\left\|\mathcal{R}^{k}\right\| \Longrightarrow\left\|\varepsilon^{k}\right\| \leq \frac{1}{\mathfrak{r}}\left\|\mathcal{R}^{k}\right\|$.

Therefore, the theorem is proved.

\section{Approximation in space: the Chebyshev collocation of the second kind}

In the current section, we get the full scheme of Eq. (6) by using the shifted Chebyshev polynomials of the second kind $\mathfrak{u}_{i}(x), i=0,1, \ldots, N$. The SCPSK is defined by the analytical structure of the Jacobi polynomials i.e. $\mathfrak{u}_{i}(x)=(i+1)\left(\begin{array}{c}i+\frac{1}{2} \\ i\end{array}\right) P_{i}^{-1} P^{\frac{1}{2}, \frac{1}{2}}(2 x-1), i=0,1, \ldots, N$ in the interval $[0,1]$ where $P_{i}^{\overline{2}, \frac{1}{2}}(x)$ is the Jacobi polynomial.

The closed-form fractional derivative for the $\eta$-order is written as

$\frac{\partial^{\eta}\left(\mathfrak{u}_{i}(x)\right)}{\partial x^{\eta}}=\sum_{k=0}^{i-\lceil\eta\rceil} \mathbf{N}_{i, k}^{\eta} \times x^{i-k-\eta}, \quad x \in[0,1], \quad i=0,1, \ldots, N$,

where $\lceil\eta\rceil$ is the ceiling of value $\eta$ and $\mathbf{N}_{i, k}^{\eta}$ is determined by

$\mathbf{N}_{i, k}^{\eta}=\frac{(-1)^{k} 4^{i-k} \Gamma(2 i-k+2) \Gamma(i-k+1)}{\Gamma(k+1) \Gamma(2 i-2 k+2) \Gamma(i-k+1-\eta)}$. 
I t $\quad \mathrm{should} \quad \mathrm{be} \quad \mathrm{stated} \quad \mathrm{that}$ $\frac{\partial^{\eta}\left(\mathfrak{u}_{i}(x)\right)}{\partial x^{\eta}}=0, i=0,1, \ldots,\lceil\eta\rceil-1, \eta>0$. Therefore, an expansion of $u\left(x, t_{j}\right)$ around the space variable is determined only by using the first $N+1$-terms of SCPSK in interval $[0,1]$ as below

$u_{N}\left(x, t_{j}\right)=\sum_{i=0}^{N} c_{i}^{j} \mathfrak{u}_{i}(x), j=0,1, \ldots, M$,

where $\left\{c_{i}^{j}\right\}_{i=0}^{N}$ is the unknown coefficients and define as follows [32]

$c_{i}^{j}=\frac{8}{\pi} \int_{0}^{1}\left(x-x^{2}\right)^{\frac{1}{2}} u_{N}\left(x, t_{j}\right) \mathfrak{u}_{i}(x) d x$.

By applying the linearity properties of the fractional derivative and substituting Eq. (12) in (11), we get the following relation

$\frac{\partial^{\eta}\left(u_{N}\left(x, t_{j}\right)\right)}{\partial x^{\eta}}=\sum_{i=\lceil\eta\rceil}^{N} \sum_{k=0}^{i-\eta} c_{i}^{j} \mathbf{N}_{i, k}^{\eta} \times x^{i-k-\eta}$.

Substituting Eqs. (12) and (14) in (6) and employing the collocation points $\left\{x_{s}\right\}_{s=1}^{N-1}$ that are the roots of SCPSK $\mathfrak{t}_{N-1}(x)$, we achieve

$\left(\lambda_{j, j}+\mathfrak{r} \delta \tau^{\alpha}\right) \sum_{i=0}^{N} c_{i}^{j} \mathfrak{t}_{i}\left(x_{s}\right)-\bar{\rho} \delta \tau^{\alpha} \sum_{i=2}^{N} \sum_{k=0}^{i-2} c_{i}^{j} N_{i, k}^{2} x_{s}^{1-k-2}-$

$\bar{\mu} \delta \tau^{\alpha} \sum_{i=1}^{N} \sum_{k=0}^{i-1} c_{i}^{j} N_{i, k}^{1} x_{s}^{i-k-1}=\sum_{m=0}^{j-1} \sum_{i=0}^{N} \bar{\lambda}_{j, m} c_{i}^{m} \mathfrak{u}_{i}\left(x_{s}\right)+\delta \tau^{\alpha} F\left(x_{s}, t_{j}\right)$,

$j=1,2, \ldots, M, \quad s=0,1, \ldots, N$,

where $c_{i}^{j}$ are the unknown coefficients. The initial solution $v_{i}^{0}$ is determined by combining relation $u(x, 0)=\psi(x)$ in Eq.

(2). To determine the boundary conditions, we can substitute Eq. (12) in (2) as below

$\sum_{i=0}^{N}(-1)^{i+2}(i+1) c_{i}^{j}=\varphi_{0}\left(t_{j}\right), \quad \sum_{i=0}^{N}(i+1) c_{i}^{j}=\varphi_{1}\left(t_{j}\right), j=1,2, \ldots, M$.

The obtained relation at Eq. (15) with the boundary conditions (16) denotes the linear algebraic system in which one can be determined the unknown coefficients $c_{i}^{i}, i=0,1,2, \ldots, N$ in each step of time $j$.

\section{Numerical investigation}

The introduced procedure of the Chebyshev collocation of the second kind in this section has a simple implementation for approaching TFBSE-EO. The numerical results in this section confirmed our claim. We then compare the achieved option prices from our scheme with those of [33] and [25] that our method gives better results. In addition, the accuracy and efficiency of the developed method demonstrated that they are obtained by the relation $\mathcal{C}_{\mathcal{O}}=\log _{2}\left(\frac{E_{i+1}}{E_{i}}\right)$ (the computational order is shown by $\mathcal{C}_{\mathcal{O}}$ ) so that $E_{i+1}$ and $E_{i}$ are errors matching to mesh size $2 M$ and $M$, respectively.

Example 1 Consider the TFBSE-EO with the exact solution $u(x, t)=(t+1)^{2} x^{2}(1-x)$

$\left\{\begin{array}{l}{ }_{0} \mathcal{D}_{t}^{\alpha} u(x, t)=\rho \frac{\partial^{2} u(x, t)}{\partial x^{2}}+\mu \frac{\partial u(x, t)}{\partial x}-r u(x, t)+f(x, t), \\ u(x, 0)=x^{2}(1-x), \quad 0<x<1, \\ u(0, t)=u(1, t)=0,\end{array}\right.$

so that $\sigma=0.25, \rho=\frac{1}{2} \sigma^{2}, \mu=r-\rho, r=0.05, \alpha=0.7$ and the source term $f(x, t)=\left(\frac{2 t^{2-\alpha}}{\Gamma(3-\alpha)}+\frac{2 t^{1-\alpha}}{\Gamma(2-\alpha)}\right) x^{2}(1-x)-$ $(t+1)^{2}\left[\rho(2-6 x)+\mu\left(2 x-3 x^{2}\right)-r x^{2}(1-x)\right]$. The boundary conditions are homogeneous. The initial condition obtains with the exact solution.

The results are demonstrated in Table 1 that hold up the theoretical results proved in Theorem 2, that is, a convergence order in time is $\mathcal{O}(2-\alpha)$ at $T=1$ with $N=7$. In Table 2, we test the presented method for the computation of the European put option with the methods of [33] and [25]. We conclude that our results are better than others with a highly low space size. In Fig. 1, we plot the approximate solution (left panel) and absolute error (right panel) at $T=1$ for $N=5$ and $M=400$.

Example 2 In this example, we consider the following TFBSE-EO with nonhomogeneous boundary conditions

$\left\{\begin{array}{l}{ }_{0} \mathcal{D}_{t}^{\alpha} u(x, t)=\rho \frac{\partial^{2} u(x, t)}{\partial x^{2}}+\mu \frac{\partial u(x, t)}{\partial x}-r u(x, t)+f(x, t), \\ u(x, 0)=x^{3}+x^{2}+1, \quad 0<x<1, \\ u(0, t)=(t+1)^{2}, \quad u(1, t)=3(t+1)^{2},\end{array}\right.$

where the dependent parameters can be considered as $\rho=1, \mu=r-\rho, r=0.5$ and $\alpha=0.7$. The source term $f(x, t)$ is achieved from the exact solution $u(x, t)=(t+1)^{2}\left(x^{3}+x^{2}+1\right)$. 
Table 1 The $L_{\infty}$ and $L_{2}$ errors and computational order with $N=7$ for Example 1 at $T=1$

\begin{tabular}{|c|c|c|c|c|c|c|c|c|}
\hline \multicolumn{5}{|l|}{$\alpha=0.3$} & \multicolumn{4}{|l|}{$\alpha=0.6$} \\
\hline$M$ & $L_{\infty}$ & $\mathcal{C}_{\mathcal{O}}$ & $L_{2}$ & $\mathcal{C}_{\mathcal{O}}$ & $L_{\infty}$ & $\mathcal{C}_{\mathcal{O}}$ & $L_{2}$ & $\mathcal{C}_{\mathcal{O}}$ \\
\hline 10 & $5.53734 \times 10^{-4}$ & & $1.19490 \times 10^{-3}$ & & $2.43144 \times 10^{-3}$ & & $5.23379 \times 10^{-3}$ & \\
\hline 20 & $1.80128 \times 10^{-4}$ & 1.62017 & $3.88797 \times 10^{-4}$ & 1.6198 & $9.47135 \times 10^{-4}$ & 1.36017 & $2.03939 \times 10^{-3}$ & 1.35972 \\
\hline 40 & $5.78236 \times 10^{-5}$ & 1.63929 & $1.24830 \times 10^{-4}$ & 1.63905 & $3.64988 \times 10^{-4}$ & 1.37572 & $7.86060 \times 10^{-4}$ & 1.37543 \\
\hline 80 & $1.83872 \times 10^{-5}$ & 1.65296 & $3.96991 \times 10^{-5}$ & 1.65279 & $1.39759 \times 10^{-4}$ & 1.38491 & $3.01033 \times 10^{-4}$ & 1.38472 \\
\hline 160 & $5.80597 \times 10^{-6}$ & 1.66309 & $1.25365 \times 10^{-5}$ & 1.66297 & $5.33099 \times 10^{-5}$ & 1.39047 & $1.14836 \times 10^{-4}$ & 1.39035 \\
\hline 320 & $1.82354 \times 10^{-6}$ & 1.6708 & $3.93772 \times 10^{-6}$ & 1.6707 & $2.02861 \times 10^{-5}$ & 1.39391 & $4.37009 \times 10^{-5}$ & 1.39384 \\
\hline $\mathcal{O}(2-\alpha)$ & & 1.7 & & 1.7 & & 1.4 & & 1.4 \\
\hline
\end{tabular}

Table 2 Comparison of error and order convergence with the methods of [33] and [25] for Example 1 at $T=1$

\begin{tabular}{|c|c|c|c|c|c|c|}
\hline \multirow[b]{3}{*}{$M$} & \multicolumn{2}{|c|}{ Method of [33] } & \multicolumn{2}{|c|}{ Method of [25] } & \multicolumn{2}{|c|}{ The current method } \\
\hline & \multicolumn{2}{|c|}{ for $N=150$ and $\alpha=0.7$} & \multicolumn{2}{|c|}{ for $N=150$ and $\alpha=0.7$} & \multicolumn{2}{|c|}{ for $N=10$ and $\alpha=0.7$} \\
\hline & $L_{\infty}$ & $\mathcal{C}_{\mathcal{O}}$ & $L_{\infty}$ & $\mathcal{C}_{\mathcal{O}}$ & $L_{\infty}$ & $\mathcal{C}_{\mathcal{O}}$ \\
\hline 10 & $3.5000 E-3$ & & $5.821 E-3$ & & $3.27707 E-3$ & \\
\hline 20 & $1.4400 E-3$ & 1.3300 & $2.304 E-3$ & 1.3372 & $1.35942 E-3$ & 1.26941 \\
\hline 40 & $5.9000 E-4$ & 1.3150 & $9.081 E-4$ & 1.3421 & $5.58865 E-4$ & 1.28242 \\
\hline 80 & $2.4000 E-4$ & 1.3400 & $3.572 E-4$ & 1.3461 & $2.28581 E-4$ & 1.28979 \\
\hline 160 & $9.5000 E-5$ & 1.3600 & $1.411 E-4$ & 1.3400 & $9.32188 E-5$ & 1.29401 \\
\hline 320 & $3.8000 E-5$ & 1.3800 & $5.387 E-5$ & 1.3892 & $3.79516 E-5$ & 1.29646 \\
\hline $\mathcal{O}(2-\alpha)$ & & 1.3 & & 1.3 & & 1.3 \\
\hline
\end{tabular}

Fig. 1 The scheme of the approximate solution (left panel) and absolute error (right panel) of Example 1 at $T=1$ for $N=5$ and $M=400$
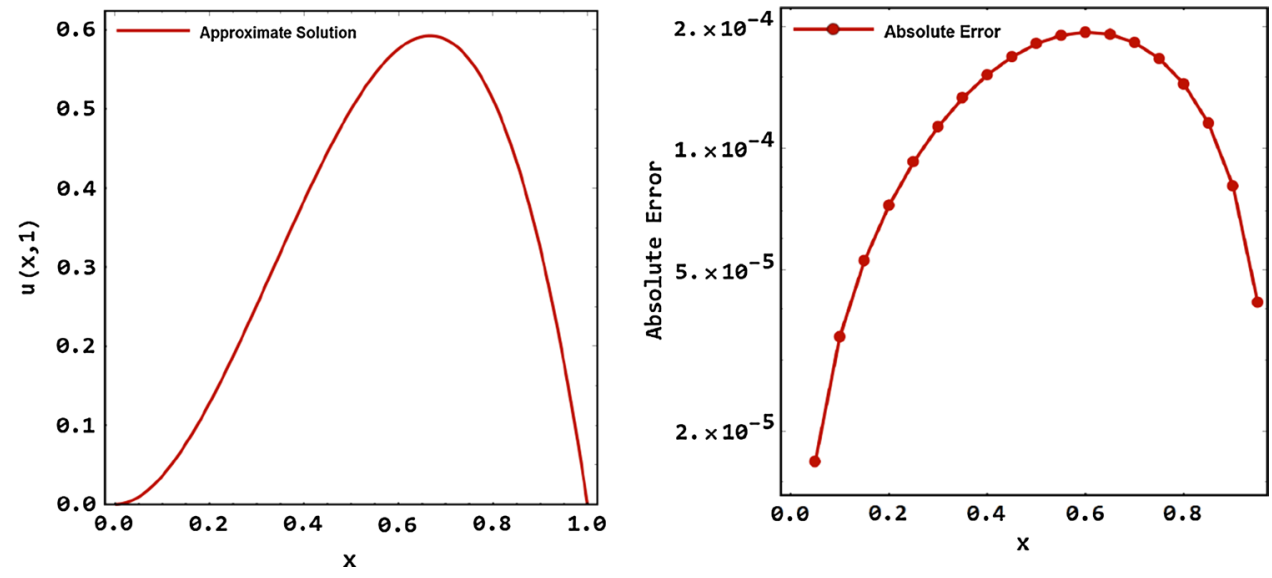

The results of this problem are presented in Tables 3, 4 . In Table 3, we obtain a convergence order that hold up the theoretical results proved in Theorem 2. In Table 4, we list comparisons between the present method with compact finite difference method [33] and radial basis functions based on finite difference scheme [25] that computational results confirm that our method gives better results.

\section{Conclusion}

The TFBSE-EO can be considered as a generalization of the classical Black-Scholes model. Due to the "globality" characteristic of the model's fractional-order derivative, the numerical solutions of this model are more complicated than the integer-order model. Accordingly, in this 
Table $3 L_{\infty}$ and $L_{2}$ error and the temporal order for Example 2 with $N=5$ at $T=1$

\begin{tabular}{|c|c|c|c|c|c|c|c|c|}
\hline \multicolumn{5}{|l|}{$\alpha=0.4$} & \multicolumn{4}{|l|}{$\alpha=0.6$} \\
\hline$M$ & $L_{\infty}$ & $\mathcal{C}_{\mathcal{O}}$ & $L_{2}$ & $\mathcal{C}_{\mathcal{O}}$ & $L_{\infty}$ & $\mathcal{C}_{\mathcal{O}}$ & $L_{2}$ & $\mathcal{C}_{\mathcal{O}}$ \\
\hline 5 & $4.14661 \times 10^{-3}$ & & $9.58912 \times 10^{-3}$ & & $9.28194 \times 10^{-3}$ & & $2.14587 \times 10^{-2}$ & \\
\hline 10 & $1.42983 \times 10^{-3}$ & 1.53609 & $3.30640 \times 10^{-3}$ & 1.53614 & $3.59235 \times 10^{-3}$ & 1.3695 & $8.30474 \times 10^{-3}$ & 1.36955 \\
\hline 20 & $4.87042 \times 10^{-4}$ & 1.55373 & $1.12623 \times 10^{-3}$ & 1.55376 & $1.37956 \times 10^{-3}$ & 1.38072 & $3.18917 \times 10^{-3}$ & 1.38075 \\
\hline 40 & $1.64493 \times 10^{-4}$ & 1.56602 & $3.80366 \times 10^{-4}$ & 1.56604 & $5.27273 \times 10^{-4}$ & 1.38758 & $1.21890 \times 10^{-3}$ & 1.38760 \\
\hline 80 & $5.52173 \times 10^{-5}$ & 1.57483 & $1.27681 \times 10^{-4}$ & 1.57484 & $2.00920 \times 10^{-4}$ & 1.39193 & $4.64465 \times 10^{-4}$ & 1.39194 \\
\hline 160 & $1.84534 \times 10^{-5}$ & 1.58124 & $4.26702 \times 10^{-5}$ & 1.58125 & $7.64133 \times 10^{-5}$ & 1.39473 & $1.76643 \times 10^{-4}$ & 1.39473 \\
\hline $\mathcal{O}(2-\alpha)$ & & 1.6 & & 1.6 & & 1.4 & & 1.4 \\
\hline
\end{tabular}

Table 4 Comparison of error and order convergence with the methods of [33] and [25] for Example 2 at $T=1$

\begin{tabular}{|c|c|c|c|c|c|c|}
\hline \multirow[b]{3}{*}{$M$} & \multicolumn{2}{|c|}{ Method of [33] } & \multicolumn{2}{|c|}{ Method of [25] } & \multicolumn{2}{|c|}{ The current method } \\
\hline & \multicolumn{2}{|c|}{ for $N=150$ and $\alpha=0.7$} & \multicolumn{2}{|c|}{ for $N=150$ and $\alpha=0.7$} & \multicolumn{2}{|c|}{ for $N=7$ and $\alpha=0.7$} \\
\hline & $L_{\infty}$ & $\mathcal{C}_{\mathcal{O}}$ & $L_{\infty}$ & $\mathcal{C}_{\mathcal{O}}$ & $L_{\infty}$ & $\mathcal{C}_{\mathcal{O}}$ \\
\hline 10 & $5.2000 E-3$ & & $6.345 E-3$ & & $4.3257 E-3$ & \\
\hline 20 & $2.0700 E-3$ & 1.3300 & $2.507 E-3$ & 1.3372 & $1.7691 E-3$ & 1.2899 \\
\hline 40 & $8.3000 E-4$ & 1.3150 & $9.957 E-4$ & 1.3421 & $7.2148 E-4$ & 1.2940 \\
\hline 80 & $3.3000 E-4$ & 1.3400 & $4.011 E-4$ & 1.3461 & $2.9376 E-4$ & 1.2963 \\
\hline 160 & $1.3000 E-4$ & 1.3600 & $1.591 E-4$ & 1.3400 & $1.1949 E-4$ & 1.2978 \\
\hline 320 & $5.0000 E-4$ & 1.3800 & $6.274 E-5$ & 1.3892 & $4.8572 E-5$ & 1.2986 \\
\hline $\mathcal{O}(2-\alpha)$ & & 1.3 & & 1.3 & & 1.3 \\
\hline
\end{tabular}

article, a numerical model to solve TFBSE-EO is proposed. First, the time discretization via the linear interpolation with the $(2-\alpha)$-order is described. Then, we explain how to obtain the approximated solution by using the Chebyshev collocation method based on the second kind. In addition, the unconditional stability of the time-discrete model is proved by using the energy method, and also it is shown that the convergence order of the time-discrete is $\mathcal{O}\left(\tau^{2-\alpha}\right)$. To demonstrate the convergence order and precision of the numerical process, we have chosen two numerical examples with exact solutions so that the numerical result has denoted the accuracy of the current model.

Open Access This article is licensed under a Creative Commons Attribution 4.0 International License, which permits use, sharing, adaptation, distribution and reproduction in any medium or format, as long as you give appropriate credit to the original author(s) and the source, provide a link to the Creative Commons licence, and indicate if changes were made. The images or other third party material in this article are included in the article's Creative Commons licence, unless indicated otherwise in a credit line to the material. If material is not included in the article's Creative Commons licence and your intended use is not permitted by statutory regulation or exceeds the permitted use, you will need to obtain permission directly from the copyright holder. To view a copy of this licence, visit http://creativecommons .org/licenses/by/4.0/.

\section{References}

1. Black, F., Scholes, M.: The pricing of options and corporate liabilities. J. Polit. Econ. 81(3), 637 (1973)

2. Merton, R.: Theory of rational option pricing. Bell J. Econom. Manage. Sci. 4, 141-183 (1973)

3. Bodie, Z., Kane, A., Marcus, A.: Investments (ISBN 0077861671) (2008)

4. Podlubny, I.: Fractional Differential Equations: An Introduction to Fractional Derivatives, Fractional Differential Equations, to Methods of their Solution and Some of their Applications. Elsevier, New York (1998)

5. Zhuang, P., Liu, F., Anh, V., Turner, I.: New solution and analytical techniques of the implicit numerical method for the anomalous subdiffusion equation. SIAM J. Numer. Anal. 46(2), 1079 (2008)

6. Zhuang, P., Liu, F., Anh, V., Turner, I.: Numerical methods for the variable-order fractional advection-diffusion equation with a nonlinear source term. SIAM J. Numer. Anal. 47(3), 1760 (2009)

7. Zhuang, P., Liu, F., Turner, I., Gu, Y.: Finite volume and finite element methods for solving a one-dimensional space-fractional Boussinesq equation. Appl. Math. Model. 38(15-16), 3860 (2014)

8. Liu, F., Zhuang, P., Turner, I., Burrage, K., Anh, V.: A new fractional finite volume method for solving the fractional diffusion equation. Appl. Math. Model. 38(15-16), 3871 (2014)

9. Zeng, F., Liu, F., Li, C., Burrage, K., Turner, I., Anh, V.: A CrankNicolson ADI spectral method for a two-dimensional Riesz space fractional nonlinear reaction-diffusion equation. SIAM J. Numer. Anal. 52(6), 2599 (2014) 
10. Zheng, M., Liu, F., Turner, I., Anh, V.: A novel high order spacetime spectral method for the time fractional Fokker-Planck equation. SIAM J. Sci. Comput. 37(2), A701 (2015)

11. Liu, Q., Liu, F., Gu, Y.T., Zhuang, P., Chen, J., Turner, I.: A meshless method based on point interpolation method (PIM) for the space fractional diffusion equation. Appl. Math. Comput. 256, 930 (2015)

12. Safdari, H., Mesgarani, H., Javidi, M., Aghdam, Y.E.: Convergence analysis of the space fractional-order diffusion equation based on the compact finite difference scheme. Comput. Appl. Math. 39(2), 1 (2020)

13. Aghdam, Y.E., Mesgrani, H., Javidi, M., Nikan, O.: A computational approach for the space-time fractional advection-diffusion equation arising in contaminant transport through porous media, Engineering With Computers (2020)

14. Nikan, O., Golbabai, A., Machado, J.T., Nikazad, T.: Numerical solution of the fractional Rayleigh-Stokes model arising in a heated generalized second-grade fluid. Eng. Comput. pp. 1-14 (2020)

15. Wyss, W.: The fractional Black-Scholes equation (2000)

16. Chen, W., Xu, X., Zhu, S.P.: Analytically pricing double barrier options based on a time-fractional Black-Scholes equation. Comput. Math. Appl. 69(12), 1407 (2015)

17. Hariharan, G., Padma, S., Pirabaharan, P.: An efficient wavelet based approximation method to time fractional Black-Scholes European option pricing problem arising in financial market. Appl. Math. Sci. 7(69), 3445 (2013)

18. Duan, J.S., Lu, L., Chen, L., An, Y.L.: Fractional model and solution for the Black-Scholes equation. Math. Methods Appl. Sci. 41(2), 697 (2018)

19. Kumar, S., Yildirim, A., Khan, Y., Jafari, H., Sayevand, K., Wei, L.: Analytical solution of fractional Black-Scholes European option pricing equation by using Laplace transform. J. Fract. Calculus Appl. 2(8), 1 (2012)

20. Mesgarani, H., Rashidnina, J., Esmaeelzade Aghdam, Y., Nikan, O.: The Impact of Chebyshev collocation method on solutions of fractional Advection-Diffusion Equation. Int. J. Appl. Comput. Math. 6(1), 1-13 (2020)

21. Safdari, H., Aghdam, Y.E., Gómez-Aguilar, J.: Shifted chebyshev collocation of the fourth kind with convergence analysis for the space-time fractional advection-diffusion equation. Eng. Comput. pp. 1-12 (2020)

22. Moghaddam, B., Dabiri, A., Lopes, A.M., Machado, J.T.: Numerical solution of mixed-type fractional functional differential equations using modified Lucas polynomials. Comput. Appl. Math. 38(2), 46 (2019)

23. Vitali, S., Castellani, G., Mainardi, F.: Time fractional cable equation and applications in neurophysiology. Chaos Solitons Fract. 102, 467 (2017)

24. Sobhani, A., Milev, M.: A numerical method for pricing discrete double barrier option by Legendre multiwavelet. J. Comput. Appl. Math. 328, 355 (2018)

25. Golbabai, A., Nikan, O., Nikazad, T.: Numerical analysis of time fractional Black-Scholes European option pricing model arising in financial market. Comput. Appl. Math. 38(4), 173 (2019)

26. Zhang, X., Sun, S., Wu, L., et al.: $\theta$-difference numerical method for solving time-fractional Black-Scholes equation. Highlights of Sciencepaper online. China Sci. Technol. Pap. 7(13), 1287 (2014)

27. Song, L., Wang, W.: In Abstract and Applied Analysis, vol. 2013 (Hindawi, 2013), vol. 2013

28. Bhowmik, S.K.: Fast and efficient numerical methods for an extended Black-Scholes model. Comput. Math. Appl. 67(3), 636 (2014)

29. Chen, W., Xu, X., Zhu, S.P.: A predictor-corrector approach for pricing American options under the finite moment log-stable model. Appl. Numer. Math. 97, 15 (2015)

30. Zhang, H., Liu, F., Turner, I., Yang, Q.: Numerical solution of the time fractional Black-Scholes model governing European options. Comput. Math. Appl. 71(9), 1772 (2016)

31. Kumar, K., Pandey, R.K., Sharma, S.: Comparative study of three numerical schemes for fractional integro-differential equations. J. Comput. Appl. Math. 315, 287 (2017)

32. Avazzadeh, Z., Heydari, M.: Chebyshev polynomials for solving two dimensional linear and nonlinear integral equations of the second kind. Comput. Appl. Math. 31(1), 127 (2012)

33. De Staelen, R.H., Hendy, A.S.: Numerically pricing double barrier options in a time-fractional Black-Scholes model. Comput. Math. Appl. 74(6), 1166 (2017) 